\title{
Determinanten des Suchverhaltens von Arbeitslosen
}

\author{
Ausgewählte Erkenntnisse basierend auf dem IZA Evaluationsdatensatz
}

\author{
Marco Caliendo • Arne Uhlendorff
}

Angenommen: 25. Januar 2011 / Online publiziert: 9. Februar 2011

(C) Institut für Arbeitsmarkt- und Berufsforschung 2011

Zusammenfassung Der Übergangsprozess von Arbeitslosigkeit in Beschäftigung wird vom Reservationslohn und den Suchanstrengungen der Arbeitslosen bestimmt. Das optimale Suchverhalten hängt dabei sowohl von institutionellen Gegebenheiten wie der Höhe und Dauer von Transferzahlungen und „klassischen“ Charakteristika wie Alter und Bildung als auch von selten beobachteten Faktoren wie Persönlichkeitseigenschaften und subjektiven Erwartungen ab. Der IZA Evaluationsdatensatz ermöglicht es, die Effekte dieser Faktoren auf das Suchverhalten bzw. den Übergangsprozess in Beschäftigung zu analysieren. Der Datensatz besteht aus einer umfangreichen administrativen $\mathrm{Zu}$ gangsstichprobe in Arbeitslosigkeit und einem Survey in dem Personen interviewt wurden, die zwischen Mitte 2007 und Mitte 2008 arbeitslos wurden. Die Arbeitslosen wurden u.a. zu ihrem Suchverhalten, zu Persönlichkeitseigenschaften, ethnischem Hintergrund und sozialen Netzwerken befragt. Unsere Analysen deuten darauf hin, dass sowohl Unterschiede in der maximalen Dauer von Transferleistungen als auch Unterschiede in Persönlichkeitseigenschaften und in subjektiven Erwartungen bezüglich der Teilnahmewahr-

M. Caliendo · A. Uhlendorff

IZA, Bonn, Deutschland

M. Caliendo

e-mail: caliendo@iza.org

M. Caliendo $\cdot$ A. Uhlendorff

DIW, Berlin, Deutschland

M. Caliendo

IAB, Nürnberg, Deutschland

A. Uhlendorff $(\varangle)$

Universität Mannheim, Mannheim, Deutschland

e-mail: uhlendorff@uni-mannheim.de scheinlichkeit an arbeitsmarktpolitischen Maßnahmen zu einer Heterogenität im Suchverhalten bzw. in der Übergangswahrscheinlichkeit in Beschäftigung führen können.

Schlüsselwörter Arbeitslosigkeitsdauer .

Arbeitslosengeld · Persönlichkeitseigenschaften ·

Subjektive Erwartungen · Evaluation

JEL Klassifikationen J64 · J65 · J68 · D84

Determinants of the search behavior of the unemployed

Selected results based on the IZA evaluation dataset

Abstract The transition process from unemployment to employment is determined by the reservation wage and the search effort of the unemployed worker. The optimal search strategy depends on labour market institutions like the generosity of unemployment benefits and "classical" characteristics like age and education as well as often not observed determinants like personality traits and subjective expectations. The IZA Evaluation dataset allows us to analyse the impact of these factors on the search behaviour and the transition process into employment. The dataset consists of a large inflow sample into unemployment based on administrative information and a unique survey of individuals who became unemployed between mid 2007 and mid 2008. This dataset allows us to observe individual job search intensity, reservation wages and job offers at different points in time as well as realised transitions into employment. Personality traits, ethnicity and social networks are part of the rich information. Our results indicate that differences in the potential duration of unemployment benefit receipt as well as differences in personality traits and in subjective expectations about participation probabilities in active labour market policy programs lead to a heterogeneity in the search behaviour and in the transition probabilities into employment. 


\section{Einleitung}

Für den Übergangsprozess von Arbeitslosigkeit in Beschäftigung sind der Reservationslohn und die Suchanstrengungen der Arbeitslosen zentrale Bestimmungsfaktoren, die auch einen Einfluss auf die individuelle Arbeitslosigkeitsdauer haben. Beide Faktoren hängen von individuellen Charakteristika - wie z.B. Alter, Bildung, und Familienstand aber auch von institutionellen Rahmenbedingungen - wie z.B. der Höhe und Dauer von Transferzahlungen - ab. Neben diesen „klassischen“ Determinanten gibt es aber auch eine Vielzahl von oftmals nicht beobachteten individuellen Eigenschaften, die auf das Suchverhalten von Arbeitslosen und damit die Arbeitslosigkeitsdauer wirken. Als zwei Beispiele seien hier (1) Antizipationseffekte von arbeitsmarktpolitischen Maßnahmen und (2) der Einfluss von Kontrolleinstellungen genannt. Der neue IZA Evaluationsdatensatz (siehe Caliendo et al. 2010b für Details) erlaubt es, eben solche Faktoren zu untersuchen. Grundlage des Datensatzes ist eine Stichprobe von Neueintritten in Arbeitslosigkeit basierend auf administrativen Daten der Bundesagentur für Arbeit, die mit einem innovativen Survey kombiniert wird. Im Survey werden ,frische“ Arbeitslose, die zwischen Mitte 2007 und Mitte 2008 arbeitslos wurden, unter anderem zu ihrem Suchverhalten, Persönlichkeitseigenschaften, ethnischem Hintergrund und sozialen Netzwerken befragt. Die erste Befragung fand dabei kurz nach dem Eintritt in Arbeitslosigkeit statt, weitere Erhebungswellen die den Arbeitsmarkterfolg der Personen ein und drei Jahr(e) nach dem Eintritt messen, sind bzw. werden zur Zeit abgeschlossen.

Erste Analysen deuten darauf hin, dass sowohl Unterschiede in der maximalen Dauer von Transferzahlungen als auch Unterschiede in Persönlichkeitseigenschaften zu einer Heterogenität im Suchverhalten bzw. in der Übergangswahrscheinlichkeit in Beschäftigung führen können. Personen mit kürzeren Anspruchsdauern auf Arbeitslosigkeit verlassen die Arbeitslosigkeit schneller. Zudem deuten die Ergebnisse auf heterogene Effekte des Bezugs von Arbeitslosengeld auf die Qualität der aufgenommenen Beschäftigung hin. Die Analyse der Effekte von Kontrolleinstellungen auf das Suchverhalten von Arbeitslosen zeigt zudem, dass Personen, die davon überzeugt sind durch eigenes Handeln die Ereignisse in ihrem Leben beeinflussen zu können, intensivere Suchanstrengungen aufweisen und einen höheren Anspruchslohn haben als Personen mit eher externaler Kontrolleinstellung. Des Weiteren zeigt sich, dass das deutsche System der Arbeitsmarktpolitik einen ,Threat-Effekt“ ausübt, d.h., dass Arbeitslose mit einer hohen individuell wahrgenommenen Teilnahmewahrscheinlichkeit an Maßnahmen der aktiven Arbeitsmarktpolitik ihren Reservationslohn senken und ihre Suchanstrengungen erhöhen.
Ziel dieses Beitrags ist, den IZA Evaluationsdatensatz sowie erste ausgewählte Ergebnisse kurz vorzustellen. ${ }^{1}$ Die oben kurz skizzierten Ergebnisse sind politikrelevant und haben direkte Implikationen für die Gestaltung von aktiver und passiver Arbeitsmarktpolitik. Es zeigt sich, dass neben „klassischen“ Determinanten von Suchverhalten auch in der Regel bisher kaum beobachtete Faktoren wie Persönlichkeitseigenschaften und subjektive Erwartungen berücksichtigt werden müssen, um eine evidenzbasierte und passgenaue Politikberatung zu ermöglichen. Wir skizzieren im Ausblick zu diesem Beitrag kurz, welche weiteren Forschungsfragen mit Hilfe des IZA Evaluationsdatensatzes untersucht werden können.

\section{IZA Evaluationsdatensatz}

Eine ausführliche Dokumentation des IZA Evaluationsdatensatzes findet sich in Caliendo et al. (2010b), wir beschränken uns hier auf eine (sehr) kurze Darstellung. Der Datensatz hat im Wesentlichen zwei Bestandteile. Der erste Teil besteht aus einer umfangreichen Zugangsstichprobe in Arbeitslosigkeit und enthält ca. 920.000 Personen die zwischen 2001 und 2008 in Arbeitslosigkeit eingetreten sind. Ein Zugang in Arbeitslosigkeit ist dabei definiert als Beginn der Registrierung als Arbeitsloser bei der Bundesagentur für Arbeit (BA). Die Datengrundlage sind die so genannten Integrierten Erwerbsbiographien (IEB), die vom Forschungsdatenzentrum der BA am Institut für Arbeitsmarkt- und Berufsforschung (IAB) bereitgestellt werden. Die IEB basieren auf administrativen Daten der Sozialversicherung für die Bestimmung von Renten- und Arbeitslosengeldansprüchen und enthalten tägliche Informationen zum Beschäftigungsstatus, Löhnen (bis zur Beitragsbemessungsgrenze), Phasen der Arbeitslosigkeit, Arbeitslosengeldbezug, Teilnahme an Maßnahmen aktiver Arbeitsmarktpolitik u.ä. (siehe Jacobebbinghaus und Seth 2007, für eine detaillierte Beschreibung der IEB). Nicht enthalten sind Informationen zu nicht sozialversicherungspflichtigen Beschäftigungszeiten wie z.B. Selbständigkeit. Die große Zahl an Beobachtungen, die detaillierten Informationen zu Beschäftigungszeiten und die lange Beobachtungsperiode sind natürlich sehr vorteilhaft. Was allerdings komplett fehlt sind potentiell wichtige Informationen z.B. über individuelle Persönlichkeitseigenschaften, Erwartungen, Suchverhalten, sozialen Netzwerken und kognitiven Fähigkeiten, die wichtig zur Erklärung des Übergangsprozesses von Arbeitslosigkeit in Beschäftigung sind. Der IZA Evaluationsdatensatz schließt

\footnotetext{
${ }^{1}$ Wir gehen in diesem Beitrag nicht auf Untersuchungen ein, die den Zusammenhang zwischen ethnischem Hintergrund und Suchverhalten betrachten, siehe hierfür bspw. Constant et al. (2009), oder den Zusammenhang zwischen sozialen Netzwerken und Suchverhalten analysieren, siehe hierfür Caliendo et al. (2010c).
} 
diese Lücke in dem eine Stichprobe von knapp 18.000 Individuen, die zwischen Mitte 2007 und Mitte 2008 in Arbeitslosigkeit eingetreten sind, kurz nach ihrem Eintritt in einem ca. 40-minütigen Interview befragt wurde. Dabei wurde neben dem Eintrittsprozess in Arbeitslosigkeit auch der Migrationshintergrund, Suchverhalten, Erwartungen über die Teilnahme an aktiver Arbeitsmarktpolitik, Persönlichkeitseigenschaften, Gesundheitszustand, Netzwerke und Präferenzen erfragt. Der Datensatz wird zu einem kurzen Paneldatensatz ausgebaut mit Wiederholungsbefragungen ein und drei Jahr(e) nach Eintritt. Für Individuen, die einer Zuspielung zustimmen, können die Survey-Informationen mit administrativen Daten der BA verknüpft werden. Der finale Datensatz steht etwa ab Herbst 2011 zur Verfügung.

\section{Arbeitslosengeldbezug und Arbeitslosigkeitsdauer ${ }^{2}$}

Die Ausgestaltung des Arbeitslosengelds (ALG) spielt eine zentrale Rolle für das Suchverhalten von Arbeitslosen. Es gibt eine Vielzahl von nationalen und internationalen empirischen Studien, die den Effekt der Länge von Unterstützungszahlungen auf die Übergangswahrscheinlichkeit von Arbeitslosigkeit in Beschäftigung analysieren (siehe bspw. Meyer 1990, und Katz und Meyer 1990, für die USA und Lalive et al. 2006, und Card et al. 2007, für Europa). Diese Studien finden in der Regel einen Anstieg der Abgangswahrscheinlichkeit aus der Arbeitslosigkeit am Ende des Transferanspruchs.

In einer früheren Studie für den deutschen Arbeitsmarkt analysiert Hunt (1995) die institutionellen Veränderungen der Bezugsdauer von Arbeitslosengeld in den 1980er Jahren. Basierend auf dem Sozio-Ökonomischen Panel (SOEP) findet sie einen negativen Effekt des Bezugs von ALG auf die Abgangswahrscheinlichkeit in Beschäftigung. Die Evidenz von nachfolgenden Studien ist allerdings gemischt; so finden beispielsweise Schneider und Hujer (1997) - ebenfalls basierend auf dem SOEP - keinen signifikanten Effekt auf die Arbeitslosigkeitsdauer. Fitzenberger und Wilke (2010) nutzen in einer jüngeren Studie administrative Daten und finden ebenfalls keinen Effekt der Reformen in den 1980er Jahren auf die Dauer zwischen dem Eintritt in Arbeitslosigkeit und der Aufnahme einer neuen Beschäftigung. ${ }^{3}$

In Caliendo et al. (2009) betrachten wir neben der Abgangswahrscheinlichkeit den Effekt der maximalen Bezugsdauer von ALG auf die Qualität des aufgenommenen

\footnotetext{
${ }^{2}$ Die Ausführungen dieses Kapitels basieren aus einer gemeinsamen Forschungsarbeit mit Konstantinos Tatsiramos. Für eine detaillierte Darstellung der Ergebnisse siehe Caliendo et al. (2009). Dieses Papier kursierte in einer vorherigen Fassung unter dem Titel „Benefit Duration, Unemployment Duration and Employment Stability: A Rregression Discontinuity Approach“.

${ }^{3}$ Für einen detaillierten Überblick über die empirischen Studien zu Deutschland siehe bspw. Fitzenberger und Wilke (2010).
}

Beschäftigungsverhältnisses, gemessen in Form der Beschäftigungsstabilität und den erzielten Löhnen. Die NichtStationarität des Suchprozesses aufgrund des zeitlich befristeten ALG-Bezugs impliziert unterschiedliche optimale Pfade von Suchanstrengungen und Reservationslöhnen für Personen mit unterschiedlichen maximalen Bezugsdauern (siehe Mortensen 1977 und van den Berg 1990). Entsprechend der Suchtheorie ist zu erwarten, dass Arbeitslose weniger in ihre Suchanstrengungen investieren und höhere Reservationslöhne wählen, je großzügiger die Arbeitslosenunterstützung ausfällt. Je näher der Zeitpunkt des Auslaufens der Arbeitslosenunterstützung rückt, desto größer ist der marginale Nutzen der Suchanstrengungen und der Aufnahme einer Beschäftigung. Dies impliziert, dass in der Periode vor dem Auslaufen des Arbeitslosengelds die Abgangswahrscheinlichkeit aus der Arbeitslosigkeit höher und die Qualität der aufgenommenen Beschäftigung geringer sind als zu Beginn der Arbeitslosigkeit. Daher betrachten wir nicht nur den durchschnittlichen Effekt, sondern analysieren auch eine mögliche Heterogenität der Effekte bezüglich der bisherigen Arbeitslosigkeitsdauer.

Unsere Identifikationsstrategie basiert auf einer Diskontinuität in der maximalen Bezugsdauer von ALG. Diese Bezugsdauer steigt in unserem Untersuchungszeitraum mit der Altersgrenze von 45 Jahren von maximal 12 auf maximal 18 Monate an. Ein Vergleich von Personen, die sich gerade unterhalb dieser Altersgrenze befinden mit Personen, die unmittelbar über dieser Grenze liegen, ergibt unter bestimmten Annahmen den kausalen Effekt einer längeren Bezugsdauer von ALG auf den Arbeitsmarktstatus. Die Analyse basiert auf dem administrativen Teil des IZA Evaluationsdatensatzes. Wir nutzen ein Sample von Neueintritten in Arbeitslosigkeit zwischen 2001 und 2003. Eine zentrale Annahme unseres Designs besteht darin, dass die Personen sich nicht in die Gruppe ober- bzw. unterhalb der Altersgrenze selektieren, indem sie bspw. den Eintritt in die Arbeitslosigkeit verzögern um von einer längeren Bezugsdauer zu profitieren. Wir finden keine Hinweise für eine entsprechende Selektion. Ein zweites mögliches Selektionsproblem besteht darin, dass wir die Qualität des Beschäftigungsverhältnisses nicht für alle Personen beobachten, sondern nur für diejenigen, die eine Beschäftigung aufgenommen haben. Obwohl die Zuweisung in die Gruppen mit 12 bzw. 18 Monaten ALG-Bezugsdauer zu Beginn der Arbeitslosigkeit lediglich durch das Alter bestimmt wird, können bei Beginn des Beschäftigungsverhältnisses aufgrund der dynamischen Selektion Unterschiede zwischen diesen beiden Gruppen in anderen beobachteten sowie in unbeobachteten Eigenschaften vorliegen (siehe Ham und LaLonde 1996, für eine ähnliche Argumentation im Kontext von sozialen Experimenten und den Effekten von Trainingsprogrammen für Arbeitslose). Daher kontrollieren wir in unserem Regressionsanalysen für beobachtbare Eigenschaften inklusive Informationen 
zur vorherigen Erwerbsbiographie als auch für eine potentielle Selektion aufgrund von unbeobachteten Eigenschaften in einem bivariaten Übergangsratenmodell.

Studien, die durchschnittliche Effekte von Transferleistungen auf die Qualität von Beschäftigungsverhältnissen analysieren finden i.d.R. keine oder nur geringe positive Effekte, siehe bspw. Card et al. (2007) für Österreich oder van Ours und Vodopivec (2008) für Slowenien. Wir bestätigen diese Ergebnisse und finden neben positiven und schwach signifikanten Effekten auf die Arbeitslosigkeitsdauer im Durchschnitt positive aber nicht signifikante Effekte auf Löhne und Beschäftigungsstabilität. Betrachten wir die Effekte in Abhängigkeit der bisherigen Arbeitslosigkeitsdauer, so zeigt sich ein Anstieg der Abgangswahrscheinlichkeit insbesondere um den Zeitpunkt des Auslaufens des ALG Bezugs (12 Monate). Außerdem finden wir Evidenz dafür, dass Arbeitslose, die kurz vor oder kurz nach dem Auslaufen des ALG-Bezugs eine Beschäftigung aufnehmen eine geringere Beschäftigungsstabilität und niedrigere Löhne aufweisen. Diese Ergebnisse sprechen dafür, dass aufgrund der Nicht-Stationarität der Arbeitsplatzsuche heterogene Effekte der ALG-Bezugsdauer nicht nur in Bezug auf die Abgangswahrscheinlichkeit sondern auch in Bezug auf die Qualität von Beschäftigungsverhältnissen existieren. Die Ergebnisse entsprechen somit den theoretischen Implikationen der Suchtheorie und deuten darauf hin, dass Arbeitslose mehr Suchanstrengungen aufweisen und weniger selektiv bezüglich der Qualität der Beschäftigung werden, je näher der Zeitpunkt des Auslaufens des Arbeitslosengeldbezugs rückt.

\section{Kontrolleinstellungen und Suchverhalten von Arbeitslosen $^{4}$}

In der klassischen Theorie der Arbeitsplatzsuche wird davon ausgegangen, dass die Arbeitslosen perfekte Informationen über die Effekte der eigenen Suchanstrengungen auf die Wahrscheinlichkeit eines Jobangebots haben. In Caliendo et al. (2010a) präsentieren wir ein alternatives Modell, in dem wir davon ausgehen, dass die Individuen eine subjektive Einschätzung über die Effektivität ihrer Suchanstrengungen haben. Diese subjektive Einschätzung hängt zumindest teilweise von der individuellen Kontrolleinstellung (,locus of control“) ab. Kontrolleinstellungen sind generalisierte Erwartungen. Weist eine Person eine internale Kontrolleinstellung auf, dann wird sie als Ursache bestimmter Handlungsfolgen in ihrem Leben vor allem die eigene Person sehen.

\footnotetext{
${ }^{4}$ Die Ausführungen dieses Kapitels basieren auf Erkenntnissen aus einer gemeinsamen Forschungsarbeit mit Deborah Cobb-Clark. Für eine detaillierte Darstellung der Ergebnisse siehe Caliendo et al. (2010a).
}

Bei externaler Kontrolleinstellung werden hingegen Faktoren außerhalb der eigenen Person als ursächlich wahrgenommen (Rotter 1966; Heckhausen 1989).

Es gibt eine Reihe von empirischen Studien, die zeigen, dass Kontrolleinstellungen mit Arbeitsmarkterfolgen korreliert sind. Ein frühes Beispiel ist Andrisani (1977, 1981), der auf Grundlage des National Longitudinal Survey einen positiven Zusammenhang zwischen einer internen Kontrolleinstellung und Löhnen feststellt. Ein Beispiel für eine Analyse mit deutschen Daten sind Anger und Heineck (2010), die auf Basis des Sozio-Ökonomischen Panels (SOEP) zeigen, dass Personen mit einer externen Kontrolleinstellung geringere Löhne aufweisen. Auch Investitionsentscheidungen scheinen mit dem Ausmaß internaler Kontrolleinstellungen zusammen zu hängen. Coleman und Deleire (2003) kommen zu dem Schluss, dass der „locus of control“ über die Erwartungen bezüglich der Renditen von Bildungsinvestitionen einen Einfluss auf die Bildungsentscheidung von Jugendlichen hat. Heckman et al. (2006) verwenden unter anderem den ,locus of control " für die Messung von nicht kognitiven Fähigkeiten und zeigen, dass diese sowohl einen Einfluss auf die Bildungsentscheidung als auch einen direkten Effekt auf die Löhne haben. Die Anzahl der Studien zum Zusammenhang vom Locus of Control und der Dauer von Arbeitslosigkeit ist begrenzt. Basierend auf Daten des SOEP zeigen Gallo et al. (2003) und Uhlendorff (2004), dass eine internale Kontrolleinstellung positiv mit einer höheren Wiederbeschäftigungswahrscheinlichkeit korreliert ist. McGee (2010) findet basierend auf dem National Longitudinal Survey of Youth Evidenz dafür, dass Kontrolleinstellungen mit dem Anspruchslohn und der Suchintensität von Arbeitslosen korreliert sind.

In Caliendo et al. (2010a) zeigen wir, dass das von uns präsentierte alternative Modell impliziert, dass Personen mit einer internalen Kontrolleinstellung höhere Anspruchslöhne haben und größere Suchanstrengungen auf sich nehmen. Diese Implikationen werden mit Hilfe der ersten Welle des Surveys des IZA Evaluationsdatensatzes getestet. Neben der im Vergleich zu Haushaltspanels wie dem SOEP oder dem BHPS großen Stichprobe von Arbeitslosen hat dieser Datensatz den Vorteil, dass alle Personen zum ungefähr gleichen Zeitpunkt nach Eintritt in die Arbeitslosigkeit (nach ca. 2 Monaten) interviewt werden. Dies reduziert das in Analysen von Zusammenhängen zwischen nicht-kognitiven Fähigkeiten und Arbeitsmarkterfolg typische Problem der potentiellen umgekehrten Kausalität zwischen Dauer in Arbeitslosigkeit und den erklärenden Faktoren.

Nach der Kontrolle für beobachtbare Unterschiede finden wir Evidenz dafür, dass Personen mit eher internaler Kontrolleinstellung sich signifikant öfter auf offene Stellen bewerben, während Personen mit externaler Kontrolleinstellung signifikant geringere Anspruchslöhne aufweisen. $\mathrm{Zu}$ dem zeigt sich, dass die subjektive Wiederbeschäftigungs- 
wahrscheinlichkeit mit zunehmenden Bewerbsanstrengungen bei internal kontrollierten Personen signifikant stärker ansteigt als bei eher external kontrollierten Arbeitslosen. Diese Ergebnisse entsprechen den Implikationen unseres theoretischen Modells.

\section{Antizipationseffekte von arbeitsmarktpolitischen Maßnahmen ${ }^{5}$}

Arbeitsmarktpolitische Maßnahmen sind weit verbreitet und zielen darauf ab, Arbeitslose (schneller) in (bessere) Beschäftigung zu bringen. Nach den jüngsten Reformen am deutschen Arbeitsmarkt ist ein implizites Ziel auch, lange Phasen in Arbeitslosigkeit durch eine schnelle und zielgenaue Zuweisung in Maßnahmen zu vermeiden. Dabei verstreicht allerdings zwischen dem Eintritt in Arbeitslosigkeit und dem Eintritt in eine Maßnahme immer eine gewisse Zeit, so dass die schlichte Existenz von AAMP-Maßnahmen schon einen Effekt auf Arbeitslose haben kann, bevor diese teilnehmen (oder von einer Teilnahme erfahren). Solche Antizipationseffekte werden schon lange diskutiert (ein frühes Beispiel ist Ashenfelter, 1978) und sind (arbeitsmarkt)politisch sehr relevant. Das optimale Suchverhalten eines Arbeitslosen wird auch dadurch bestimmt, mit welcher Wahrscheinlichkeit er von einer Teilnahme in bestimmten Programmen ausgeht. Rechnet er z.B. mit hoher Wahrscheinlichkeit damit in Kürze an einem Trainingsprogramm teilzunehmen das ihm hilft sich effizienter (mit geringeren Kosten) zu bewerben, könnte es für ihn optimal sein weniger intensiv zu suchen und den Reservationslohn zu erhöhen. In Evaluationsstudien ist es oft von Interesse ob eine AAMPMaßnahme die Arbeitslosigkeitsdauer im ersten Jahr nach Eintritt reduzieren kann. Dabei wird nur selten analysiert, ob dieser Effekt durch ex-post oder ex-ante Effekte von Programmen zustande kommt. Ist z.B. der ex-post Effekt einer Maßnahme positiv (die Trainingsmaßnahme hilft dem Arbeitslosen „bessere“ Bewerbungen zu schreiben und so schneller eine Anstellung zu finden) der ex-ante Effekt aber negativ (das Programm wurde erst zu einem späten Zeitpunkt des Arbeitslosigkeitsspells angeboten, so dass der Arbeitslose zu lange in Arbeitslosigkeit verharrt ist) könnte der ermittelte Gesamteffekt gleich null sein.

Ex-ante Effekte sind üblicherweise schwer zu identifizieren. Van den Berg et al. (2009) nutzen subjektiv empfundene Teilnahmewahrscheinlichkeiten von ,frischen“ Arbeitslosen und vergleichen die Suchanstrengungen von Personen mit einer hohen Teilnahmewahrscheinlichkeit mit denen mit einer niedrigen Teilnahmewahrscheinlichkeit. Dabei

${ }^{5}$ Die Ausführungen dieses Kapitels basieren auf Van den Berg et al. (2009). zeigt sich, dass Personen mit einer hohen empfundenen Teilnahmewahrscheinlichkeit ihren Reservationslohn um knapp 3\% senken und ihre Suchanstrengungen (gemessen in der Anzahl der genutzten Suchkanäle) intensivieren. Insgesamt kann also festgehalten werden, dass das System der deutschen Arbeitsmarktpolitik eine abschreckende Wirkung hat, die Arbeitslose dazu veranlasst, stärker zu suchen. Bergemann et al. (2010) zeigen zudem, dass diese Effekte mit dem Migrationshintergrund der arbeitslosen Individuen variieren. In zukünftigen Analysen muss untersucht werden, inwieweit die Erwartungen der Individuen mit tatsächlich realisierten Maßnahmen übereinstimmen und welchen Einfluss die individuell erwarteten Effekte der Maßnahmen auf das Suchverhalten haben.

\section{Zusammenfassung und Ausblick}

Dieser kurze Beitrag hat deutlich gemacht, dass der Übergangsprozess von Arbeitslosigkeit in Beschäftigung nicht nur von „klassischen“ Faktoren und institutionellen Rahmenbedingungen abhängt, sondern auch von Persönlichkeitseigenschaften und individuellen Erwartungen. Unsere Ergebnisse zeigen, dass Personen mit kürzeren Anspruchsdauern auf Arbeitslosigkeit die Arbeitslosigkeit schneller verlassen, deuten aber auch auf heterogene Effekte des Bezugs von Arbeitslosengeld auf die Qualität der aufgenommenen Beschäftigung hin. Die Analyse der Effekte von Kontrolleinstellungen auf das Suchverhalten von Arbeitslosen zeigt, dass Personen, die davon überzeugt sind durch eigenes Handeln die Ereignisse in ihrem Leben beeinflussen zu können, intensivere Suchanstrengungen aufweisen und einen höheren Anspruchslohn haben als Personen mit eher externaler Kontrolleinstellung. Darüber hinaus senken Arbeitslose mit einer hohen subjektiv wahrgenommenen Teilnahmewahrscheinlichkeit an Maßnahmen der aktiven Arbeitsmarktpolitik ihren Reservationslohn und erhöhen ihre Suchanstrengungen.

Ziel einer evidenzbasierten Arbeitsmarktpolitik muss es sein, neben den Effekten von institutionellen Faktoren in Zukunft auch den Einfluss von bisher kaum beachteten Dimensionen wie Persönlichkeitseigenschaften oder subjektiven Erwartungen zu berücksichtigen. Hier besteht allerdings noch weiterer Forschungsbedarf, wofür der IZA Evaluationsdatensatz eine geeignete Datengrundlage bietet. Eine arbeitsmarktpolitisch relevante Forschungsfrage ist beispielsweise, inwieweit mit Persönlichkeitseigenschaften wie Kontrolleinstellungen heterogene Effekte von Instrumenten der aktiven Arbeitsmarktpolitik wie dem Monitoring von Bewerbungsaktivitäten oder der Teilnahme an Fortbildungsmaßnahmen einhergehen. Derartige Erkenntnisse könnten für eine zielgruppenspezifische und effiziente Verwendung knapper Ressourcen in der Arbeitsmarktpolitik von großer 
Bedeutung sein. Der IZA Evaluationsdatensatz erlaubt hierbei nicht nur die Analyse von Effekten auf das Suchverhalten, sondern durch Ausnutzung der Panelstruktur auch die Untersuchung von mittelfristigen Effekten auf Beschäftigungswahrscheinlichkeiten und Löhne.

\section{Executive Summary}

In this research project we analyze the transition process from unemployment to employment. Thereby we focus on the determinants of reservation wages and the type and intensity of job search as well as their impact on unemployment duration. Both components depend on factors like education, age, unemployment benefits and household composition. Next to these "classical" variables the process may depend on factors like personality traits or social networks, which are usually unobservable for the econometrician. The IZA Evaluation dataset allows us to analyse the impact of these factors on the search behaviour and the transition process into employment. The dataset consists of a large inflow sample into unemployment based on administrative information and a unique survey of individuals who became unemployed between mid 2007 and mid 2008. This dataset allows us to observe individual job search intensity, reservation wages and job offers at different points in time as well as realized transitions into employment.

Based on administrative data, we use a sharp discontinuity in the maximum benefit duration to identify the effect of extended duration on unemployment duration and post-unemployment outcomes. We find a spike in the reemployment hazard for the unemployed workers with shorter benefit duration. We also show that those unemployed who obtain jobs close to and after the time when benefits are exhausted are significantly more likely to exit subsequent employment and receive lower wages.

Based on survey data we estimate the impact of locus of control, i.e., the extent to which a person believes that future outcomes are determined by his or her own actions as opposed to external factors, on job search behavior. We find evidence that individuals with an internal locus of control search more and that individuals who believe that their future outcomes are determined by external factors have lower reservation wages.

Labor market programs may affect unemployed individuals' behavior before they enroll. Such ex ante effects are hard to identify without model assumptions. We develop a novel method that relates selfreported perceived treatment rates and job-search behavioral outcomes to each other. To deal with effect heterogeneity and selectivity, the effects of interest are estimated by propensity score matching. We apply the method to the German ALMP system, using a novel data set including self-reported assessments of the variables of interest as well as an unusually detailed amount of information on behavior, attitudes, and past outcomes. We find that the system generates a negative ex ante effect on the reservation wage and a positive effect on search effort. We additionally show that the effects vary with respect to ethnicity.

Danksagung Die Autoren danken einem anonymen Gutachter für hilfreiche Hinweise und der Deutschen Forschungsgemeinschaft (DFG) für finanzielle Unterstützung im Rahmen des Schwerpunktprogramms „Flexibilisierungspotenziale bei heterogenen Arbeitsmärkten“, Teilprojekt CA 829/1-1.

\section{Literatur}

Andrisani, P.J.: Inter-external attitudes, personal initiative, and the labor market experience of black and white men. J. Hum. Resour. 12, 308-328 (1977)

Andrisani, P.J.: Internal-external attitudes, personal initiative, and the labor market experience: a reply to Duncan and Morgan. J. Hum. Resour. 16, 658-666 (1981)

Anger, S., Heineck, G.: The returns to cognitive abilities and personality traits in Germany. Labour Econ. 17, 535-546 (2010)

Bergemann, A., Caliendo, M., Van den Berg, G.J.: The effect of active labor market programs on not-yet treated unemployed individuals. J. Eur. Econ. Assoc. 7, 606-616 (2009)

Bergemann, A., Caliendo, M., Van den Berg, G.J., Zimmermann, K.F.: The threat effect of participation in active labor market programs on job search behavior of migrants in Germany. IZA discussion paper 5400, Bonn (2010)

Caliendo, M., Cobb-Clark, D., Uhlendorff, A.: Locus of control and job search strategies. IZA discussion paper 4750, Bonn (2010a)

Caliendo, M., Falk, A., Kaiser, L., Schneider, H., Uhlendorff, A., Van den Berg, G.J., Zimmermann, K.F.: The IZA evaluation dataset: towards evidence-based labour policy-making. IZA discussion paper, Bonn (2010b)

Caliendo, M., Schmidl, R., Uhlendorff, A.: Social networks, job search methods and reservation wages: evidence for Germany. IZA discussion paper 5165, Bonn (2010c)

Caliendo, M., Tatsiramos, K., Uhlendorff, A.: Benefit duration, unemployment duration and job match quality: a regressiondiscontinuity approach. IZA discussion paper 4670, Bonn (2009)

Card, D., Chetty, R., Weber, A.: Cash-on-hand and competing models of intertemporal behavior: new evidence from the labor market. Q. J. Econ. 122, 1511-1 (2007)

Coleman, M., Deleire, T.: An economic model of locus of control and the human capital investment decision. J. Hum. Resour. 38, 701721 (2003)

Constant, A.F., Kahanec, M., Rinne, U., Zimmermann, K.F.: Ethnicity, job search and labor market reintegration of the unemployed. IZA discussion paper 4660 (2009)

Fitzenberger, B., Wilke, R.A.: Unemployment durations in WestGermany before and after the reform of the unemployment compensation system during the 1980s. Ger. Econ. Rev. 11, 336-366 (2010)

Gallo, W.T., Endrass, J., Bradly, E.H., Hell, D., Kasl, S.V.: The influence of internal control on the employment status of German workers. Schmollers Jahrb. Wirtsch.- Soz.wiss. 123, 71-82 (2003)

Ham, J., LaLonde, R.: The effect of sample selection and initial conditions in duration models: evidence from experimental data on training. Econometrica 64, 175-205 (1996)

Heckhausen, H.: Motivation und Handeln. 2. Aufl. Springer, Berlin/ Heidelberg/New York (1989) 
Heckman, J.J., Stixrud, J., Urzua, S.: The effects of cognitive and noncognitive abilities on labor market outcomes and social behavior. J. Labor Econ. 24, 411-482 (2006)

Hunt, J.: The effect of the unemployment compensation on unemployment duration in Germany. J. Labor Econ. 13, 88-120 (1995)

Jacobebbinghaus, P., Seth, S.: The German integrated employment biographies sample IEBS. Schmollers Jahrb. Wirtsch.- Soz.wiss. 127, 335-342 (2007)

Katz, L.F., Meyer, B.D.: The impact of the potential duration of unemployment benefits on the duration of unemployment. J. Public Econ. 41, 45-72 (1990)

Lalive, R., van Ours, J.C., Zweimüller, J.: How changes in financial incentives affect the duration of unemployment. Rev. Econ. Stud. 73, 1009-1038 (2006)

McGee, A.: How the perception of control influences unemployed jobsearch. Working paper (2010)

Meyer, B.D.: Unemployment insurance and unemployment spells. Econometrica 58, 757-782 (1990)

Mortensen, D.: Unemployment insurance and job search decisions. Ind. Labor Relat. Rev. 30, 505-517 (1977)

Van den Berg, G.J.: Nonstationarity in job search theory. Rev. Econ. Stud. 57, 255-277 (1990)

Van den Berg, G.J., Bergemann, A., Caliendo, M.: The effect of active labor market programs on not-yet treated unemployed individuals. J. Eur. Econ. Assoc. 7, 606-616 (2009)

Van Ours, J., Vodopivec, M.: Does reducing unemployment insurance generosity reduce job match quality? J. Public Econ. 92, 684-695 (2008)

Rotter, J.: Generalized expectancies for internal versus external control of reinforcement. Psychol. Monogr. 80, 1-28 (1966)
Schneider, H., Hujer, R.: Wirkungen der Unterstützungsleistungen auf die Arbeitslosigkeitsdauer in der Bundesrepublik Deutschland: Eine Analyse der Querschnitts und Längsschnittdimension. In: Hujer, R., Rendtel, U., Wagner, G. (Hrsg.) Wirtschafts- und Sozialwissenschaftliche Panel-Studien, Datenstrukturen und Analyseverfahren. Sonderhefte zum Allgemeinen Statistischen Archiv, Bd. 30, S. 71-88. Vandenhoeck and Ruprecht, Göttingen (1997)

Uhlendorff, A.: Der Einfluss von Persönlichkeitseigenschaften und sozialen Ressourcen auf die Arbeitslosigkeitsdauer. Köln. Z. Soziol. Soz.psychol. 56, 279-303 (2004)

Marco Caliendo ist seit 2009 Forschungsdirektor am Forschungsinstitut zur Zukunft der Arbeit (IZA) in Bonn. Davor war er als Senior Research Associate am IZA (2007-2009) und DIW Berlin (2005-2007) beschäftigt. Forschungsfelder: Evaluation arbeitsmarktpolitischer Maßnahmen, Selbstständigkeit/Entrepreneurship, Einfluss von Persönlichkeitseigenschaften auf ökonomische Ergebnisse, angewandte Mikroökonometrie

Arne Uhlendorff is currently a research associate at the University of Mannheim. He studied economics and sociology at the University of Cologne and graduated in October 2002. From November 2002 till July 2007 he was a research associate at the DIW Berlin and finished his doctoral studies in economics at the Free University Berlin. From August 2007 until September 2009 he was a Research Associate at IZA. His research interests include labor economics and applied microeconometrics, with a focus on the evaluation of labor market policies, employment dynamics, job search behavior and duration analysis. 